\title{
Circulating levels of receptor activator of nuclear factor-kB ligand/osteoprotegerin/macrophage-colony stimulating factor in a presumably healthy human population
}

\author{
Svetlana Trofimov ${ }^{1}$, Ia Pantsulaia ${ }^{1,2}$, Eugene Kobyliansky ${ }^{1}$ and Gregory Livshits ${ }^{1}$ \\ ${ }^{1}$ Sackler Faculty of Medicine, Tel Aviv University, Israel and ${ }^{2}$ Department of Biomedicine, Institute of Medical Biotechnology, \\ Georgian Academy of Sciences, Georgia
}

(Correspondence should be addressed to G Livshits, Human Population Biology Research Unit, Department of Anatomy and Anthropology, Sackler Faculty of Medicine, Tel Aviv University, Ramat Aviv 69978, Tel Aviv, Israel; Email: gregl@post.tau.ac.il)

\begin{abstract}
Objectives: To determine the ranges of variation of circulating receptor activator of nuclear factor- $\mathrm{B}$ ligand (RANKL)/osteoprotegerin (OPG)/macrophage-colony stimulating factor (M-CSF) and to ascertain their potential relationships with age, sex and menopausal status in women, and with sex hormones in a population-based healthy cohort.

Subjects and methods: Blood samples were collected with EDTA after an overnight fast. The plasma levels of each of the above biochemical indices were measured by ELISA in a total of 566 apparently healthy individuals aged $18-75$ years.

Results: The plasma concentrations of cytokine molecules in the entire sample ranged from 674 to $4929 \mathrm{pg} / \mathrm{ml}$ for OPG, from 105 to $4468 \mathrm{pg} / \mathrm{ml}$ for soluble RANKL (sRANKL), and from 187 to $7604 \mathrm{pg} / \mathrm{ml}$ for M-CSF. The OPG levels demonstrated a clear positive correlation with age in both sexes $(r=0.42$ and $0.43, P<0.001$, for men and women respectively). Application of the two-interval mathematical model revealed that in females OPG levels were age-independent until age 42, but then showed clear and significant correlation with age $(r=0.48, P<0.001)$. As a result, young females (before 42 years) had a substantially lower average OPG level, $1377.8 \pm 327.68 \mathrm{pg} / \mathrm{ml}$, in comparison with older women, $1666.02 \pm 397.14 \mathrm{pg} / \mathrm{ml}$. The M-CSF correlation with age was significantly greater in women $(r=0.29, P<0.001)$ compared with men $(r=0.17, P<0.01)$. Significant negative correlations between plasma levels of both OPG and M-CSF with estradiol concentrations were observed in women $(r=-0.39, P<0.01 ; r=-0.25, P<0.001$ respectively). sRANKL did not correlate with either age or sex hormones in either women or men.

Conclusion: Age and sex affect differently the interindividual variation of OPG, RANKL and M-CSF. Our observations could form the basis for further research to establish provisional reference limits for OPG and RANKL, which are potential markers for benign and malignant processes in bone.
\end{abstract}

European Journal of Endocrinology 150 305-311

\section{Introduction}

Tumor necrosis factor (TNF) superfamily molecules, namely, the receptor activator of nuclear factor- $\mathrm{kB}$ ligand (RANKL), its receptor (RANK), and its soluble (decoy) receptor, osteoprotegerin (OPG), comprise a novel cytokine system exerting pleiotropic effects on bone metabolism, the immune system and endocrine functions $(1,2)$. RANKL is expressed on the osteoblast/stromal cell surface, binds to its receptor (RANK) on the surface of hematopoietic precursor cells, and in the presence of macrophage-colony stimulating factor (M-CSF), stimulates osteoclastogenesis. OPG, a secreted glycoprotein of the TNF receptor superfamily, acts as a non-signaling decoy receptor that binds RANKL and prevents the activation of RANK. A recent ultrastructural study (3) on the precise roles of RANKL and OPG in the differentiation and resorptive functions of osteoclasts (OCs) has shown that: (i) RANKL and M-CSF exert different effects on preOC differentiation through modulation of the cellular phenotype; (ii) RANKL and OPG are important regulators of not only the terminal differentiation of OCs but also their resorptive function; and (iii) the binding of M-CSF and RANKL to their respective receptors is conceivably a necessary and sufficient condition to initiate osteoclastogenesis.

Recent findings indicate that the RANKL/RANK/OPG system is implicated in various skeletal and immunemediated diseases characterized by increased bone resorption and bone loss, including several forms 
of osteoporosis (postmenopausal, glucocorticoidinduced and senile osteoporosis) (2), bone metastases (4), periodontal disease (5) and rheumatoid arthritis (6). Whereas a relative deficiency of OPG has been found to be associated with osteoporosis in various animal models (2), in postmenopausal women the parenteral administration of OPG $(3 \mathrm{mg} / \mathrm{kg})$ rapidly reduced enhanced biochemical markers of bone turnover by $30-80 \%$ (7). These investigations have clearly established the RANKL/OPG system as a key cytokine network involved in the regulation of bone cell biology, OC-OC and bone-immune cross talks, and in the maintenance of bone mass.

This not withstanding, it is well known that the bone resorption pathway is influenced by a variety of osteotropic factors, including sex hormones. In vitro studies have demonstrated that estrogen boosts OPG production and inhibits RANKL expression and the responsiveness of OC precursors, whereas testosterone (TESTO) and the non-aromatizable androgen $5 \alpha$-dihydrotestosterone appear to exert an opposite effect $(8-10)$. In vivo data concerning the relationship between menopause, bone loss and RANKL/OPG levels in humans are still equivocal. For example, Khosla's team (11) did not detect in women any significant correlation between serum OPG levels and estradiol (ESTR) concentrations, while in men they tended to be inversely correlated with the bioavailable ESTR levels. Contrariwise to these findings, Szulc et al.'s group (12) reported in men weak positive correlations between serum OPG levels, free TESTO and free ESTR indices. Studies designed to assess OPG serum levels in aging women and men with or without osteoporosis have yielded contradictory results $(13-15)$. OPG has also been administered as a therapeutic agent, resulting in a dramatic reduction in bone turnover, but little is known about its long-term effect on bone density (7).

From all the above, it is clear that the clinical significance of RANKL/OPG circulating levels has just recently started to emerge $(4,6,15,16)$, and more work needs to be done, particularly to assess local (bone microenvironment) and systemic levels (plasma, serum) of OPG, RANKL and M-CSF. Thus, the main aim of the present study was to determine circulating levels of OPG, RANKL and M-CSF, and to ascertain their potential relationships with age, sex and menopausal status in women, and with sex hormones (ESTR, TESTO) in a population-based healthy cohort.

\section{Subjects and methods}

Our cohort consisted of 566 healthy individuals, specifically 289 men and 277 women, aged $19-75$ years and belonging to 126 households. The studied population was ethnically Caucasian (Chuvasha, Russian Federation) and characterized by a demographically stable structure with traditional relationships between family members. These villagers have lived at least for the last few generations under the same environmental conditions. The gathered demographic information entailed gender, age, education, occupation, information on physical activity, reproductive histories, and data on chronic morbidity and medical treatment. Individuals with known bone disease or risk factors for increased bone mineral density (BMD) loss (such as steroid hormone therapy, diabetes and hyperparathyroidism) were not included in the study. A more detailed description of the chosen population is given by us elsewhere (17). This study was conducted in line with the declaration of Helsinki with the approval of the Tel Aviv University Ethics Committee. Written informed consent was obtained from all participants.

\section{Hormonal and biochemical marker measurements}

The plasma samples were collected after an overnight fast $(10-12 \mathrm{~h})$ into tubes with EDTA and the cells were removed by centrifugation at $1800 \boldsymbol{g}(15 \mathrm{~min})$ and stored in aliquots at $-70^{\circ} \mathrm{C}$ until analyzed. These were gathered in 1999, and the cytokines were measured during 2001-2002. For this period of this time the samples remained frozen without repeat thaw-freeze cycles. The concentrations of OPG were measured by a sandwich ELISA using the set of specific antibodies and standards from $R \& D$ Systems (Minneapolis, MN, USA). MaxiSorp plates (Nunc Inc., Roskilde, Denmark) were coated overnight at $4^{\circ} \mathrm{C}$ with anti-human monoclonal OPG antibody. Unbound antibodies were washed away using $0.2 \%$ Tween 20 in PBS. Then, the plates were blocked with $1 \%$ BSA in PBS for $1 \mathrm{~h}$ at room temperature. After washing, the samples or recombinant human $\mathrm{OPG} / \mathrm{Fc}$ chimera were added and incubated for $1 \mathrm{~h}$ at room temperature. According to the manufacturer's protocol, the detecting biotinylated goat IgG anti-human OPG (for monomer and dimer) antibody was added and the plates again incubated for $2 \mathrm{~h}$ at room temperature. Next, streptavidin conjugated to horseradish peroxidase was added and the plates incubated for $20 \mathrm{~min}$ at room temperature. The optical density was measured at $450 \mathrm{~nm}$ with correction on $570 \mathrm{~nm}$ using an automatic ELISA reader (ELX808 Ultra Microplate Reader; Bio-tek Instruments, Inc., Winooski, Vermont, USA). The range of the assay of OPG was $62.5-4000 \mathrm{pg} / \mathrm{ml}$. The minimum detectable dose was determined by adding two standard deviations to the mean optical density value of the zero standard replicates and calculating the corresponding concentration. The results were calculated using a four-parameter curve fit and expressed as $\mathrm{pg} / \mathrm{ml}$. The detection limit was $40 \mathrm{pg} / \mathrm{ml}$. The mean of intra- and interassay coefficients of variation were 6 and $8 \%$ respectively.

Soluble RANKL (sRANKL) was also measured by a set of specific antibodies (for detection of free RANKL) and standards of the same company (R\&D Systems). 
The measurement principle and the test procedure of this assay correspond to that described above for OPG. The concentrations were calculated based on a recombinant human RANKL standard. The assay performance was characterized by a lower detection limit of $20 \mathrm{pg} / \mathrm{ml}$ and the mean of intra- and interassay coefficients of variation were 8 and $10 \%$ respectively. In both cases, for minimizing the interassay coefficients of variation we used only one lot of reagents to measure all plasma samples. Those results differing by more than $20 \%$ were re-assayed. Standards were freshly prepared before the assay.

For the quantitative determination of M-CSF plasma level a commercial Quantikine ELISA kit (R\&D Systems) was applied. Prior to the assay, all samples were diluted 1:5 into the Calibrator Diluent RD6F (R\&D Systems). Standards and samples were pipetted into the wells and all M-CSF present in the plasma was bound by the immobilized antibody. After unbound compounds were washed away, an enzyme-linked polyclonal antibody specific for M-CSF was added to the wells. Following a wash to remove any unbound antibody-enzyme reagent, a substrate solution was added to the wells and color developed in proportion to the amount of M-CSF bound in the initial step. The color reaction was stopped and the intensity of the color was measured. Results are expressed as $\mathrm{pg} / \mathrm{ml}$. The lower detection limit of this assay was $9 \mathrm{pg} / \mathrm{ml}$. The mean intra- and interassay coefficients of variation were 4 and $6 \%$ respectively.

Also measured were the plasma levels of the sex hormones (TESTO and ESTR). Total TESTO and ESTR values were determined by means of standard RIA procedures using TESTO-CT2 and ESTR-US-CT RIA kits (CIS Bio International, ORIS Group, Gif-Sur-Yvette, Cedex, France) as previously described (18).

\section{BMD measurements}

BMD was measured using plane X-ray radiographs of the middle and distal phalanges of the third finger of both hands, with an aluminum wedge as control (17). Roentgenographic densitometry of hand bones is a precise procedure that has a coefficient of variation ranging from 0.6 to $2 \%$, and an accuracy error about $4 \%$ (19); it conforms well with BMD in other parts of the skeleton and is suitable for use under field conditions.

\section{Statistical analysis}

Analyses were performed by means of the STATISTICA 5.5/PC statistical package (Statsoft, Inc., Tulsa, OK, USA). Values of OPG, RANKL, M-CSF and sex hormones were not normally distributed and therefore were logtransformed before analysis. Testing the distribution of each of the studied cytokines it was obvious that some values should be considered as outlying, because they were inconsistent with the distribution properties of the bulk of the data. To ensure that some rare observations were not eventually excluded from the data, we used $\geq 4$ S.D. as a criterion (and not 3 S.D.). All outliers were re-assayed, and only after that were they excluded from further analysis (20 individuals for RANKL, 11 for OPG and 3 for M-CSF).

Correlations between plasma levels of OPG, sRANKL, M-CSF and sex hormones as well as between them and radiographic hand BMD were examined using Pearson's correlation with and without adjustment for age and body mass index (BMI) and in relation to sex. To make our data comparable with those reported by others $(13,20)$, men and women were divided into two age groups: $\leq 50$ years; $>50$ years. In women, these age groups coincided with pre- and postmenopausal status. However, to ascertain the possible agedependence of each cytokine, several piecewise linear models were examined. To choose the best-fitting curve, maximum likelihood of the parameter estimates for different statistical models were obtained using the software CURVEF (21). In fact, two models were tested, namely, a linear model and a two-interval model. The latter model divides the age curve into two parts. For each part (age interval) two parameters are introduced into the model: parameter $\mathrm{T}_{\mathrm{i}}$, estimating the age at which the given part of the age-dependence begins, and parameter $\beta_{\mathrm{i}}$, estimating the rate of change per year. Thus, in the absence of age-related changes $\beta_{i}=0$, whereas in the absence of significant change in the pattern of age-dependence (i.e. linear correlation) $\mathrm{T}_{\mathrm{i}}=0$.

\section{Results}

Table 1 provides basic descriptive statistics on the studied biochemical variables and on body weight and height, according to sex and in two age cohorts (below and above 50 years). The data for sRANKL/OPG/M-CSF system cytokines and sex steroids are presented before log-transformation and in the original units. Males above 50 years of age showed a slight but non-significant tendency toward decrease in the sex steroid levels. In postmenopausal women, however, there was a profound decline in the sex steroid levels compared with the premenopausal women.

The plasma concentrations of cytokine molecules in the entire sample ranged from 674 to $4929 \mathrm{pg} / \mathrm{ml}$ for OPG, from 105 to $4468 \mathrm{pg} / \mathrm{ml}$ for sRANKL, and from 187 to $7604 \mathrm{pg} / \mathrm{ml}$ for M-CSF. The mean concentrations of sRANKL and M-CSF were significantly different in men and women (Table 1); however, there were no significant sex differences in OPG circulating levels $(P=0.48)$. When two age groups (below and above 50 years) were compared within the gender, we found that OPG and M-CSF differed significantly in men and women (Mann-Whitney U test, with significance ranging from $P=0.05$ for M-CSF in men to $P<0.001$ for OPG in women). sRANKL levels 
Table 1 Descriptive statistics (means \pm S.D. with ranges) for men and women in two age cohorts (younger and older than 50 years).

\begin{tabular}{|c|c|c|c|c|}
\hline & \multicolumn{2}{|c|}{ Men } & \multicolumn{2}{|c|}{ Women } \\
\hline & $<50$ years $n=163$ & $\geq 50$ years $n=126$ & Premenopausal $n=165$ & Postmenopausal $n=112$ \\
\hline Age (years) & $31.77 \pm 8.07(18.00-49.00)$ & $61.76 \pm 6.61(5.00-75.00)$ & $33.29 \pm 9.47(18.00-49.00)$ & $62.01 \pm 6.79(50.00-75.00)$ \\
\hline Weight $(\mathrm{kg})$ & $64.97 \pm 10.34(46.20-100.10)$ & $64.53 \pm 12.98(41.00-100.30)$ & $57.9 \pm 10.68(37.60-95.80)$ & $63.38 \pm 11.92(38.10-97.50)$ \\
\hline Height $(\mathrm{m})$ & $1.69 \pm 0.06(1.59-1.89)$ & $1.63 \pm 0.05(1.40-1.80)$ & $1.56 \pm 0.05(1.42-1.78)$ & $1.51 \pm 0.05(1.39-1.65)$ \\
\hline $\mathrm{BMI}\left(\mathrm{kg} / \mathrm{m}^{2}\right)$ & $22.78 \pm 3.13(16.38-32.76)$ & $24.09 \pm 4.23(16.20-36.40)$ & $23.66 \pm 4.91(15.78-39.77)$ & $27.84 \pm 4.94(16.58-44.39)$ \\
\hline TESTO (nmol/l) & $16.55 \pm 6.1(0.60-37.50)$ & $14.78 \pm 7.53(0.70-38.70)$ & $1.70 \pm 4.37(0.30-34.90)$ & $1.83 \pm 4.29(0.10-17.10)$ \\
\hline ESTR $(\mathrm{pmol} / \mathrm{l})$ & $63.22 \pm 42.42(16.80-308.10)$ & $62.61 \pm 42.5(0.10-223.30)$ & $340.15 \pm 516.34(24.20-779.20)$ & $69.77 \pm 144.53(0.70-595.20)$ \\
\hline $\mathrm{OPG}^{1,2}(\mathrm{pg} / \mathrm{ml})$ & $1430.7 \pm 408.3(674.20-2576.10)$ & $1778.74 \pm 478.7(724.00-2947.20)$ & $1378.5 \pm 321.6(685.00-2856.40)$ & $1759.4 \pm 380.3(961.90-2958.40)$ \\
\hline $\mathrm{M}-\mathrm{CSF}^{1,2}(\mathrm{pg} / \mathrm{ml})$ & $857.5 \pm 379.9(187.30-2014.60)$ & $949.3 \pm 375.8(248.90-2251.60)$ & $706.1 \pm 325.4(222.30-1961.30)$ & $970.8 \pm 455.5(302.50-2011.50)$ \\
\hline sRANKL $^{1,2}(\mathrm{pg} / \mathrm{ml})$ & $1096.2 \pm 793.6(115.40-3402.30)$ & $948.26 \pm 719.10(120.10-3111.60)$ & $1249.8 \pm 855.6(104.90-3495.20)$ & $1175.8 \pm 921.8(122.20-3461.80)$ \\
\hline
\end{tabular}

${ }^{1} P$ values for comparisons of gender averages (Mann-Whitney $U$ test): $P=0.48$ (for OPG); $P=0.004$ (for M-CSF) and $P=0.008$ (for sRANKL)

${ }^{2} P$ values for comparisons of age cohorts in men and women (Mann-Whitney $\mathrm{U}$ test): $P<0.0001, P<0.0001$ (for OPG); $P=0.033, P<0.0001$ (for M-CSF) and $P=0.135, P=0.076$ (for sRANKL).
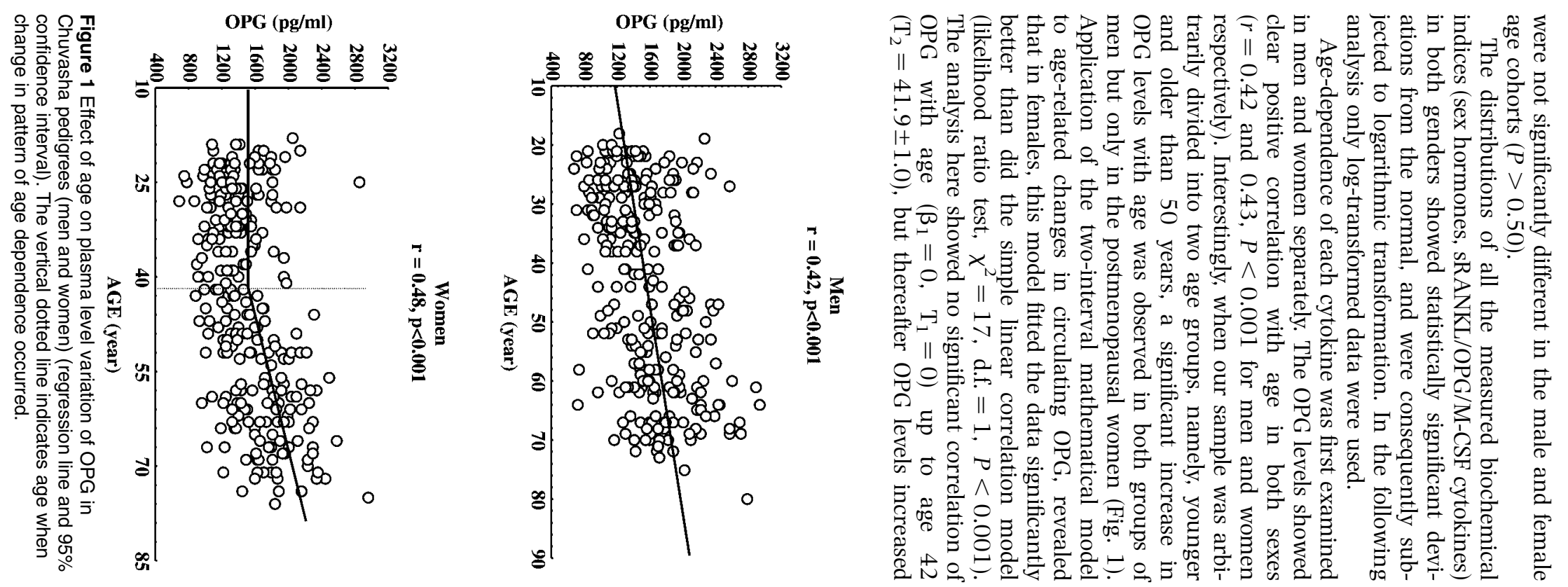
sharply $\left(\beta_{2}=(10 \pm 1)^{-4}\right)$ with correlation $r=0.48$ $(P<0.001)$ in this age cohort (Fig. 1). However, OPG changes in men, and M-CSF changes in men and women, demonstrated a simple linear correlation. The extent of M-CSF correlation with age was substantially greater in women $(r=0.29, P<0.001)$ compared with men $(r=0.17, P=0.012)$. Constraining both correlations to equal each other was significantly $(P<0.001)$ rejected by a maximum likelihood ratio test as implemented in statistical package FISHER (http://hpcio.cit.nih.gov/lserver/FISHER.html).

sRANKL levels did not correlate significantly with age.

Next, we examined correlations between circulating cytokines and sex hormones. Reliable negative associations between plasma levels of OPG and M-CSF with ESTR concentrations were observed in women $(r=-0.39, P<0.001 ; r=-0.25, P<0.011$ respectively), but none of the sex hormones correlated with cytokines in men. Neither did sRANKL correlate with any of the studied cytokines or sex hormones in women or men. OPG levels were inversely associated with hand BMD in women $(r=-0.26, P<0.001)$ but not in men. However, after adjusting for age, the correlation between them became statistically insignificant $(P=0.590)$. Plasma sRANKL levels were not correlated with BMD.

We also tested whether the age-adjusted levels of OPG, sRANKL and M-CSF correlated with one another but found that only circulating M-CSF was weakly associated with OPG in the total sample of men and women. The associations between the other studied molecules were not significant. Note also that in the subsamples of premenopausal women there was significant correlation between M-CSF and SRANKL ( $r=0.25, P=0.012)$, yet, in the young men cohort $(<50)$, correlation between these two markers was negative $(r=-0.22, P=0.02)$. No other significant correlations were found in the total sample or in the sex/age subsamples.

\section{Discussion}

Cell-to-cell interaction between osteoblasts/stromal cells and preOCs is essential for OC formation, and OPG and RANKL coordinately regulate bone density and structure by a well-balanced regulation of OC differentiation from hematopoietic precursors $(1,2$, 22 ). On the other hand, M-CSF produced by osteoblastic cells has long been known to induce OC differentiation from hematopoietic cells and to stimulate their function and survival (23). Although the findings suggested an important role for OPG/sRANKL/M-CSF as a local and paracrine factor, it still remains unclear whether OPG/sRANKL/M-CSF circulates in the blood and if so, whether the circulatory OPG/sRANKL/MCSF ratio plays any role in bone physiology, especially in diseases characterized by excessive bone resorption.
In the present study, we report the characterization of circulating OPG, sRANKL and M-CSF levels in an apparently healthy cohort of men and women. We further note that the sRANKL and M-CSF plasma mean values were significantly higher in women than in men, while the OPG levels did not differ between the genders, but were significantly lower in premenopausal women than in postmenopausal women $(P<0.001)$ and in younger men $($ age $<50)$ than in older ones $(P<0.001)$.

Consistent with findings from other studies $(11,13$, 20), we have demonstrated an age- but not a sexdependent increase of OPG levels in both men and women (Fig. 1). Interestingly, judging from our data and those of Yano et al. (13) and Ziolkowska et al. (20), it would seem that the OPG concentrations increase after about age 50 in the respective Chuvasha, Japanese and Polish populations. Hence, when analyzing OPG levels, a clear demarcation between the young and elderly is strongly recommended. An important question deserving an answer is what agedependent factor(s) regulate(s) plasma OPG levels. At present, regrettably, there is no clear information about either the regulatory mechanism or the primary source of circulating OPG. Given the important changes in sex hormone levels that occur at about age 50 in both men and women, it has been suggested that OPG production in a healthy population may be influenced by these hormones $(9,12)$. For instance, there is evidence from in vitro studies that ESTR stimulates the expression of OPG (24), while in vivo studies have evinced a positive correlation between serum OPG and ESTR levels in both sexes $(12,14)$. Khosla and colleagues (9) demonstrated that ESTR tended to increase and TESTO to decrease (in the presence of an aromatase inhibitor) circulating OPG levels in men. Surprisingly, serum OPG levels were not correlated with ESTR levels in women and, in fact, tended to be inversely associated with bioavailable ESTR levels in men (11). In the present study, we found that circulating ESTR levels in women correlated negatively with OPG levels $(r=-0.35, P<0.001)$, but in men OPG was not related to sex hormone levels. Recently, Kudlacek et al. (25) also reported a weak inverse correlation between ESTR and OPG levels in healthy women $(r=-0.16, P<0.001)$. The reasons for this dissimilarity in results between these findings and other in vitro studies in cell systems and in vivo work are unclear. They may stem from the different OPG and sRANKL assays used in different investigations. The second possibility may be that we assessed total circulatory levels of ESTR and TESTO, while the bioavailable fractions of sex steroids can enter in the cell and be biologically active. Another reason may be that we measured OPG levels only in the peripheral circulation, and it is unclear to what extent this reflects actual changes in the bone microenvironment. Yet, according to Ueland et al. (26), OPG is produced in many tissues, 
including bone, whence it may be released to the circulation, so that the serum levels also reflect the local milieu within the bone. The authors detected an agerelated increase of OPG levels in cortical and trabecular bone matrix in postmenopausal women; moreover, these changes were inversely correlated with BMD, possibly providing a link to the pathophysiology of postmenopausal osteoporosis. Our results are in good agreement with this study in that radiographic hand BMD decreased significantly with increase in OPG levels in women. Finally, we supposed that other agerelated factors could also influence plasma OPG levels.

There are grounds for believing that the biological activity of OPG as well as of sex hormones is dependent on the relative levels of both sRANKL and M-CSF (27). Data concerning the behavior of serum concentrations of sRANKL in healthy subjects and under pathological conditions are still lacking. The secretion of sRANKL has been considered to signal pathological processes, e.g. bone resorption in hypercalcemia of malignancy (28). While OPG was gender-independent, the different sRANKL concentrations and consequently the ratios of sRANKL to OPG between male and female are obvious. The comparison of the RANKL/OPG ratio in our data $(0.77 \pm 0.65)$ with published data on healthy subjects (29-31), which also used the ELISA assay, revealed that our results are in good agreement with these studies.

As mentioned in the Introduction, M-CSF is an additional crucial cytokine for OC formation $(2,23)$, and its circulatory levels have been reported to be elevated in patients with various diseases (32). In the present study, however, apparently healthy subjects were examined. The M-CSF levels in our sample were within the range of healthy individuals in other populations and in accordance with previous observations showed significant correlation with age in both men and women cohorts $(33,34)$. Moreover, in women, M-CSF plasma concentrations had a highly significantly negative correlation with circulating ESTR $(P<0.001)$. This finding conforms well with Kamada et al.'s (33) data that hormone replacement therapy induced a significant $(P<0.01)$ increase in serum $M$ CSF levels in postmenopausal women. It is of interest to note that our present analysis revealed a highly significant $(P<0.001)$ correlation between $\mathrm{M}-\mathrm{CSF}$ and OPG, both in men and in women. M-CSF and sRANKL plasma levels also appeared to be interrelated in young $(<50)$ men and women, albeit the later correlation had a negative sign. In view of the potential clinical importance of the studied cytokines as biomarkers of several diseases, it is obvious that further research is needed for elucidation of the gender differences in the possible influence of M-CSF and OPG/sRANKL system components on bone metabolism and certain functions of the immune system. However, whatever the reason for this phenomenon, the appreciation of gender-dependent reference ranges for RANKL and other cytokines seems to be necessary.

\section{Acknowledgements}

This study was supported jointly by the Israel National Science Foundation to G L (Grant No. 544/00-1) and by a Postdoctoral Fellowship Grant (I P) kindly provided by UNESCO and Israel (the Ministry of Education, the Council for Higher Education's Planning and Budgeting Committee, the Ministry of Foreign Affairs and Israel National Commission for UNESCO). We also thank two anonymous reviewers for their constructive comments and suggestions on an early draft of the manuscript.

\section{References}

1 Walsh MC \& Choi Y. Biology of the TRANCE axis. Cytokine and Growth Factor Reviews 200314 51-63.

2 Hofbauer L, Khosla S, Dunstan C, Lacey L, Boyle W \& Riggs B. The roles of osteoprotegerin and osteoprotegerin ligand in the paracrine regulation of bone resorption. Journal of Bone and Mineral Research $2000152-12$.

3 Ross F \& Teitelbaum S. Osteoclast biology. In Osteoporosis, pp 73-105. Eds D Feldman \& J Kelsey. San Diego: Academic Press, 2001.

4 Brown J, Vessella R, Kostenuik P, Dunstan C, Lange P \& Corey E. Serum osteoprotegerin levels are increased in patients with advanced prostate cancer. Clinical Cancer Research 200110 977-983.

5 Teng Y, Nguyen H, Gao X, Kong Y, Gorczynski R, Singh B et al. Functional human T-cell immunity, and osteoprotegerin ligand control alveolar bone destruction in periodontal infection. Journal of Clinical Investigation $2000 \mathbf{1 0 6}$ R59-R67.

6 Feuerherm A, Borset M, Seidel C, Sundan A, Leistad L, Ostensen M et al. Elevated levels of osteoprotegerin (OPG) and hepatocyte growth factor (HGF) in rheumatoid arthritis. Scandinavian Journal of Rheumatology $200130229-234$.

7 Bekker P, Holloway D, Nakanishi A, Arrighi M, Leese P \& Dunstan C. The effect of a single dose of osteoprotegerin in postmenopausal women. Journal of Bone and Mineral Research 2001 $16348-360$.

8 Hofbauer L, Hicok K, Chen D \& Khosla S. Regulation of osteoprotegerin production by androgens and anti-androgens in human osteoblastic lineage cells. European Journal of Endocrinology $2002147269-273$.

9 Khosla S, Atkinson EJ, Dunstan C \& O'Fallon W. Effect of estrogen versus testosterone on circulating osteoprotegerin and other cytokine levels in normal elderly men. Journal of Clinical Endocrinology and Metabolism 200287 1550-1554.

10 Bord S, Ireland D, Beavan S \& Compston J. The effects of estrogen on osteoprotegerin, RANKL, and estrogen receptor expression in human osteoblasts. Bone 200332 136-141.

11 Khosla S, Arrighi H, Melton LJ III, Atkinson E, O'Fallon W, Dunstan C et al. Correlates of osteoprotegerin levels in women and men. Osteoporosis International 200213 394-399.

12 Szulc P, Hofbauer LC, Heufelder AE, Roth S \& Delmas P. Osteoprotegerin serum levels in men: correlation with age, estrogen, and testosterone status. Journal of Clinical Endocrinology and Metabolism $2001863162-3165$.

13 Yano K, Tsuda E, Washida N, Kobayashi F, Goto M, Harada A et al. Immunological characterization of circulating osteoprotegerin/ osteoclastogenesis inhibitory factor: increased serum concentrations in postmenopausal women with osteoporosis. Journal of Bone and Mineral Research 199914 518-527.

14 Rogers A, Saleh G, Hannon RA, Greenfield D \& Eastell R. Circulating estradiol and osteoprotegerin as determinants of bone turnover and bone density in postmenopausal women. Journal of Clinical Endocrinology and Metabolism $2002 \quad \mathbf{8 7}$ $4470-4475$. 
15 Fahrleitner-Pammer A, Dobnig H, Piswanger-Soelkner C, Bonelli C, Dimai HP, Leb G et al. Osteoprotegerin serum levels in women: correlation with age, bone mass, bone turnover and fracture status. Wiener Klinische Wochenschrift $2003 \mathbf{1 1 5}$ $291-297$.

16 Jung K, Lein M, Hosslin K, Grosse A, Roth S, Possinger K et al. Osteoprotegerin and receptor activator of nuclear factor-kappaB ligand (RANKL) in the serum of healthy adults. International Journal of Biological Markers 200217 177-181.

17 Livshits G, Karasik D \& Kobyliansky E. Complex segregation analysis of the radiographic phalanges bone mineral density and their age-related changes. Journal of Bone and Mineral Research $200217152-161$.

18 Livshits G, Yakovenko C \& Kobyliansky E. Quantitative genetic analysis of circulating levels of biochemical markers of bone formation. American Journal of Medical Genetics 200094 324-331.

19 Sartoris DJ. Quantitative bone mineral analysis. In Bone and Joint Imaging, edn 2, pp 154-164. Ed. D Resnick. Philadelphia, PA: WB Saunders Co., 1996.

20 Ziolkowska M, Kurowska M, Radzikowska A, Luszczykiewicz G, Wiland P, Dziewczopolski W et al. High levels of osteoprotegerin and soluble receptor activator of nuclear factor kappa B ligand in serum of rheumatoid arthritis patients and their normalization after anti-tumor necrosis factor alpha treatment. Arthritis and Rheumatism $2002 \mathbf{4 6} 1744-1753$.

21 Malkin I, Karasik D, Livshits G \& Kobyliansky E. Modelling of agerelated bone loss using cross-sectional data. Annals of Human Biology $200229256-270$.

22 Suda T, Kobayashi K, Jimi E, Udagawa N \& Takahashi N. The molecular basis of osteoclast differentiation and activation. Novartis Foundation Symposium 2001232 247-250.

23 Quinn J, Elliott J, Gillespie M \& Martin T. A combination of osteoclast differentiation factor and macrophage-colony stimulating factor is sufficient for both human and mouse osteoclast formation in vitro. Endocrinology $19981394424-4427$.

24 Saika M, Inoue D, Kido S \& Matsumoto T. 17beta-estradiol stimulates expression of osteoprotegerin by a mouse stromal cell line, ST-2, via estrogen receptor-alpha. Endocrinology 2001142 2205-2212.

25 Kudlacek S, Schneider B, Woloszczuk W, Pietschmann P \& Willvonseder R. Serum levels of osteoprotegerin increase with age in a healthy adult population. Bone $200332681-686$.
26 Ueland T, Bollerslev J, Godang K, Muller F, Froland S \& Aukrust P. Increased serum osteoprotegerin in disorders characterized by persistent immune activation or glucocorticoid excess - possible role in bone homeostasis. European Journal of Endocrinology 2001145 685-690.

27 Shiotani A, Takami M, Itoh K, Shibasaki Y \& Sasaki T. Regulation of osteoclast differentiation and function by receptor activator of NF-кB ligand and osteoprotegerin. Anatomical Record 2002268 137-146.

28 Nagai M, Kyakumoto S \& Sato N. Cancer cells responsible for humoral hypercalcemia express mRNA encoding a secreted form of ODF/TRANCE that induces osteoclast formation. Biochemical and Biophysical Research Communications 2000269 532-536.

29 Alvarez L, Peris P, Guanabens N, Vidal S, Ros I, Pons F et al. Serum osteoprotegerin and its ligand in Paget's disease of bone: relationship to disease activity and effect of treatment with bisphosphonates. Arthritis and Rheumatism $2003 \mathbf{4 8} 824-828$.

30 Terpos E, Szydlo R, Apperley JF, Hatjiharissi E, Politou M, Meletis J et al. Soluble receptor activator of nuclear factor $\kappa \mathrm{B}$ ligand-osteoprotegerin ratio predicts survival in multiple myeloma: proposal for a novel prognostic index. Blood 2003102 1064-1069.

31 Jung K, Lein M, Hösslin K, Grosse A, Roth S, Possinger K et al. Osteoprotegerin and receptor activator of nuclear factor- $\mathrm{kB}$ ligand in serum of healthy adults. International Journal of Biological Markers 200217 177-181.

32 Motoyoshi K. Biological activities and clinical application of M-CSF. International Journal of Hematology 199867 109-122.

33 Kamada M, Irahara M, Maegawa M, Ohmoto Y, Takeji T, Yasui T et al. Postmenopausal changes in serum cytokine levels and hormone replacement therapy. American Journal of Obstetrics and Gynecology 2001184 309-314.

34 Hasegawa Y, Sawada M, Ozaki N, Inagaki T \& Suzumura A. Increased soluble tumor necrosis factor receptor levels in the serum of elderly people. Gerontology $2000 \mathbf{4 6}$ 185-188.

Received 21 May 2003

Accepted 21 November 2003 